VOL. $3(1970), 49-54$.

\title{
Regular metabelian groups of prime-power order
}

\author{
R. J. Faudree
}

\begin{abstract}
Let $H$ be a finite metabelian $p$-group which is nilpotent of class $c$. In this paper we will prove that for any prime $p>2$ there exists a finite metacyclic $p$-group $G$ which is nilpotent of class $c$ such that $H$ is isomorphic to a section of a finite direct product of $G$.
\end{abstract}

\section{Introduction}

Subgroups and factor groups of regular groups are regular but the direct product of two regular groups is not necessarily regular (see [5]). Moreover the variety generated by all finite regular $p$-groups for $p>3$ is the variety of all groups (see [2]) and the variety generated by all finite metabelian regular $p$-groups for $p>2$ is the variety of all metabelian groups (see [4]). The main result of this paper extends some of the results mentioned above by showing for $p>2$ that not only do you get irregular p-groups by taking finite direct products of regular p-groups but every finite metabelian p-group can be obtained by taking factor groups of subgroups of finite direct products of finite metabelian regular p-groups.

\section{Proof of theorem}

In the remaining discussion we will assume $p>2$ and $H$ is a fixed finite metabelian $p$-group of exponent $p^{e}$ which is nilpotent of class $c$. For any positive integer $r$ let

Received 28 March 1970. 


$$
G_{r}=\left\langle a, b: a^{p^{r c}}=1, b^{p^{c}}=1 \text {, and } a^{-1} b^{-1} a b=a^{p^{r}}\right\rangle
$$

$G_{r}$ is a metacyclic group which is nilpotent of class $c$ and of exponent $p^{r c} \cdot G_{r}$ is also regular (see [1]).

We will prove

THEOREM. $H$ is in the variety generated by $G_{r}$ for $r$ sufficiently Zarge.

A well-known property of finite groups in the variety generated by a finite group (see [3]) gives the following:

COROLLARY. $B$ is isomorphic to a section of a finite direct product of $G_{r}$ for sufficiently large $r$.

Let $X_{\infty}$ denote the free group of countable rank on generators $\left\{x_{1}, x_{2}, \ldots\right\}$. Let $g$ be an element of $X_{\infty} \cdot g$ is a simple commutator in normal form of weight $v$, weight $g=v$, and sign $u$, sign $g=u$ and involving precisely $\left\{x_{1}, \ldots, x_{t}\right\}$ if

$$
g\left(x_{1}, x_{2}, \ldots, x_{t}\right)=\left(x_{i(1)}, x_{i(2)}, \ldots, x_{i(v)}\right)
$$

where $i(1)=1, i(2)=u, i(j) \leq i(k)$ if $2<j \leq k$ and $\{i(1), \ldots, i(v)\}=\{1, \ldots, t\} . d_{j}(g)$ will denote the number of occurrences of $x_{j}$ in $g$.

An element $f$ of $X_{\infty}$ is in normal form of weight $\leq c$ if

$$
f=\prod_{i=1}^{i} f_{i}^{\gamma_{i}}
$$

where the $f_{i}$ are distinct simple commutators in normal form of weight $\leq c$ involving precisely $\left\{x_{1}, \ldots, x_{t}\right\}, Z$ is an arbitrary positive integer, $t$ is a positive integer $\leq c$, and the $\gamma_{i}$ are non-zero integers. Let $L_{r}$ denote the words of $X_{\infty}$ which are in normal form of weight $\leq c$ and are laws of $G_{r}$. A basis for the laws of $G_{r}$ is 
$L_{p} \cup\left\{\left(\left(x_{1}, x_{2}\right),\left(x_{3}, x_{4}\right)\right),\left(x_{1}, \ldots, x_{c+1}\right)\right\}$, (see [6]). Therefore to prove the theorem it is sufficient to prove:

PROPOSITION. For sufficiently large $r, f$ in $L_{r}$ implies $p^{e}$ divides $\gamma_{i}(1 \leq i \leq l)$.

Before we can complete the proof of the proposition it will be necessary to state and prove some elementary lemas.

For $m$ a positive integer let $\theta(m)$ be the highest power of $p$ dividing $m !$.

LEMMA 1 .

i) If $m=\sum_{i=0}^{t} k_{i} p^{i}$ with $0 \leq k_{i} \leq p-1$ then

$$
\theta(m)=\left(m-\sum_{i=0}^{t} k_{i}\right) /(p-1) ;
$$

ii) for positive integers $n$ and $m, p^{\theta(m)}$ divides $(m+n) ! / n !$.

Proof. The number of positive multiples of $p^{i},(1 \leq i \leq t)$, less than or equal to $m$ is $\sum_{j=i}^{t} k_{j} p^{j-i}$. Thus

$$
\theta(m)=\sum_{i=1}^{t} \sum_{j=i}^{t} k_{j} p^{j-i}=\left(m-\sum_{i=0}^{t} k_{i}\right) /(p-1) .
$$

ii) is a consequence of the fact that $m$ ! divides $(m+n) ! / n !$..

Let $z$ denote the integers, $R=z\left[y_{1}, \ldots, y_{t}\right]$ the polynomial ring over $z$ in indeterminates $y_{1}, \ldots, y_{t}$, and $J_{n}$ the ideal of $R$ generated by $p^{n}$ for $n$ a non-negative integer. Denote $z /\left(z \cap J_{n}\right)$ by $z_{n}$

LEMMA 2. Let $h=h\left(y_{1}, \ldots, y_{t}\right)$ be an element of $R$ such that the degree in each variable is $\leq c$ and tc $<n$. If $h$, considered as 
a function of $Z$ into $Z$, has only values in $J_{n} \cap Z$ then $h$ is in $J_{m}$ for $m=n-t \theta(c)$.

Proof. Let $t=1$. We can assume that $y_{1}=y$ and $h$ is a polynomial of degree $c$. Since $h(1)$ is in $J_{n} \cap Z$

$$
h(y)=(y-1) h_{1}(y)+h_{1}^{\prime}(y)
$$

where $h_{1}^{\prime}$ is a polynomial in $J_{n}$. Assume for $j \leq c-1$ that

$$
\text { * } \quad h(y)=\left(\prod_{i=1}^{j}(y-i)\right) h_{j}(y)+h_{j}^{\prime}(y)
$$

where $h_{j}^{\prime}$ is a polynomial in $J_{m}$ which as a function has only values in $J_{n}$. Thus $(j !) h_{j}(j+1)$ is in $J_{n} \cap Z$ and

$$
h_{j}(y)=(y-j-1) \cdot h_{j+1}(y)+k(y)
$$

for $k(y)$ in $J_{n-\theta(j)}$

$$
h_{j+1}^{\prime}(y)=\prod_{i=1}^{j}(y-i) \cdot k(y)+h_{j}^{\prime}(y)
$$

has the same properties as $h_{j}^{\prime}(y)$. Therefore * is true for $1 \leq j \leq c$.

Since $h(y)$ has degree $c$

$$
h(y)=\prod_{i=1}^{c}(y-i) \cdot b+h_{c}(y)
$$

where $b$ is in 2 . Hence $(c !) b$ is in $J_{n} \cap Z, b$ is in $J_{m} \cap Z$ and $h$ is in $J_{m}$. Induction on $t$ will complete the proof.

For non-negative integers $i \leq j$ let $\left(\begin{array}{l}j \\ i\end{array}\right)=j ! /((j-i) ! i !)$. For any positive integer $r$ let $\sigma_{r}$ be the function on the non-negative integers defined by 


$$
\sigma_{r}(j)=\sum_{i=1}^{j}\left(\begin{array}{l}
j \\
i
\end{array}\right) p^{r(i-1)}
$$

For any positive integer $n, \sigma_{r}$ induces a map $\sigma_{r}^{\prime}$ of $z_{n}$ into $z_{n}$.

LEMMA 3. $\sigma_{r}^{\prime}$ is onto.

Proof. It suffices to show that $\sigma_{r}^{\prime}$ is injective. Assume $\sigma_{p}(k)-\sigma_{p}(j)$ is in $z \cap J_{n}$ for $0 \leq j<k<p^{n}$. Clearly $k-j$ is in $Z \cap J_{s}$ for $s=\min \{n, r\} . k-j$ in $Z \cap J_{Z r}(Z \geq 1)$ implies $p^{r(i-1)}\left(\left(\begin{array}{l}k \\ i\end{array}\right)-\left(\begin{array}{l}j \\ i\end{array}\right)\right\}$ is in $z \cap J_{r(l+1)}$ if $2 \leq i \leq j$ and $\left(\begin{array}{l}k \\ i\end{array}\right) p^{r(i-1)}$ is in $J_{r(z+1)}$ if $i>j$. Hence $k-j$ is in $z \cap J_{s}$ for $s=\min \{(z+1) x, n\}$. Induction on $z$ gives that $k-j$ is in $z \cap J_{n}$. Proof of the proposition. Let $f=\prod_{i=1}^{l} f_{i}^{\gamma_{i}}$ be an element of $L_{r}$. We can assume $f$ involves precisely $\left\{x_{1}, \ldots, x_{t}\right\}$ and $\gamma_{i}=\beta_{i} p^{\alpha}$ $(I \leq i \leq 2)$ with $B_{i}$ relatively prime to $p$. Let $u$ be a fixed integer between 2 and $t, n_{i j}=d_{j}\left(f_{i}\right)-\delta_{j u}(I \leq i \leq l, 1 \leq j \leq t)$ where $\delta_{j u}$ is the Kronecker delta, and $w_{i}=\sum_{j=1}^{t} n_{i j}$.

For any function $j$ of $z$ into $z$ define the homomorphism $\tau_{j}$ of $x_{\infty}$ into $G_{p}$ by $\tau_{j}\left(x_{k}\right)=b^{j(k)}$ if $k \neq u$ and $\tau_{j}\left(x_{k}\right)=a^{-1} b^{j(k)}$ if $k=u$. By assumption $\tau_{j}(f)=1$ for all $j$. Also $\tau_{j}\left(f_{i}\right)=1$ for all $j$ if $\operatorname{sign} f_{i} \neq u$. If $\operatorname{sign} f_{i}=u$ then $\tau_{j}(f)=a^{\phi(i)}$ where $\phi(i)=p^{r w_{i}} \prod_{k=1}^{t}\left(\sigma_{r}(j(k))\right)^{n} i k$. If only the first $s$ of the $f_{i}$ have sign $u$ then

$$
\sum_{i=1}^{s} \beta_{i} p^{r w_{i}+\alpha_{i}}\left(\int_{k=1}^{t} \sigma_{r}(j(k))^{n i k}\right)
$$


is in $J_{r e} \cap Z$. By Lemma 3 the only values of the polynomial

$$
h\left(y_{1}, \ldots, y_{t}\right)=\sum_{i=1}^{s} \beta_{i} p^{m w_{i}+\alpha}\left(_{k=1}^{t} y_{k}{ }^{n}\right)
$$

considered as a function are in $J_{r_{c}} \cap Z$. Lemma 2 implies $w w_{i}+\alpha_{i} \geq \operatorname{rct} t \theta(c) \quad(1 \leq i \leq s)$. Thus

$$
\alpha_{i} \geq r\left(c-w_{i}\right)-t \theta(c)
$$

$w_{i}<c$ and $t \leq c$ since each $f_{i}$ involves precisely $\left\{x_{1}, \ldots, x_{t}\right\}$. The above argument is true for any $u$. Therefore $\alpha_{i} \geq e \quad(1 \leq i \leq 2)$ if $r$ is sufficiently large.

\section{References}

[1] P. Hall, "On a theorem of Frobenius", Proc. London Math. Soc. (2) 40 (1936), 468-501.

[2] P. Hall, "A note on $\overline{S I-g r o u p s ", ~ J . ~ L o n d o n ~ M a t h . ~ S o c . ~} 39$ (1964), 338-344.

[3] Graham Higman, "Some remarks on varieties of groups", Quart. J. Math. oxford Ser. (2) 10 (1959), 165-178.

[4] I.D. Macdonald, "The variety of regular p-groups", Arch. Math. 18 $(1967), 359-361$.

[5] Paul M. Weichsel, "Regular p-groups and varieties", Math. 2. 95 (1967), 223-231.

[6] Paul M. Weichsel, "On metabelian p-groups", J. Austral. Math. Soc. 7 $(1967), 55-63$.

University of IIIinois at Urbana-Champaign,

Urbana, IIIinois, USA. 